\title{
Evaluación de la eficiencia del gasto social en los países EU15 con análisis envolvente de datos y métodos cluster borrosos
}

\author{
DE ANDRÉS SÁNCHEZ, JORGE \\ Cátedra de Inclusión Social Social \& Business Research Laboratory (SBRlab) \\ Universidad Rovira i Virgili \\ Correo electrónico: jorge.deandres@urv.cat \\ Belzunegui ERAso, Ángel Gabriel \\ Cátedra de Inclusión Social Social \& Business Research Laboratory (SBRlab) \\ Universidad Rovira i Virgili \\ Correo electrónico: angel.belzunegui@urv.cat \\ VALLS FONAYET, FRANCESC \\ Cátedra de Inclusión Social Social \& Business Research Laboratory (SBRlab) \\ Universidad Rovira i Virgili \\ Correo electrónico: francesc.valls@urv.cat
}

\begin{abstract}
RESUMEN
En el estudio de los sistemas de bienestar la relación entre el gasto social y los indicadores de vulnerabilidad como la tasa de pobreza o índices de desigualdad de ingresos tienen gran interés en la literatura. Este trabajo evalúa la productividad del gasto social de los estados de la EU15 (los estados de bienestar más consolidados de la EU28), en el período 2011-2015, con metodología Análisis Envolvente de Datos. Posteriormente, con un método clustering difuso identificamos los patrones existentes de gasto social y su eficiencia. Observamos tres grupos de países. El primero engloba la mayor parte de estado del bienestar nórdico y continental. El segundo grupo, conformado por Luxemburgo e Irlanda, son países con el menor volumen de gastos social sobre PIB pero a la vez son países eficientes. El tercero engloba a los estados del bienestar mediterráneos, junto con Gran Bretaña, que son los menos eficientes en la reducción de los indicadores de vulnerabilidad.
\end{abstract}

Palabras clave: desigualdad de rentas, pobreza, frontera eficiente, análisis envolvente de datos, clustering borroso.

Clasificación JEL: C49; F02; H50; H53.

MSC2010: 03E72; 62A86; 90C08. 


\title{
Evaluation of the efficiency of social spending in EU15 countries with data envelopment analysis and fuzzy clustering methods
}

\begin{abstract}
In the study of welfare systems, the relation of the social expenditure and the indicators of vulnerability as poverty rates or income inequality indexes focus a great interest in the literature. In this way, we evaluate the productivity of social transfer policies of EU15 states, which are the countries with more consolidated Welfare States within EU28, during the period 2011-2015, with data envelopment analysis. Subsequently, with a fuzzy clustering method we identify the existing patterns of social expenditures and their efficiency. We identify three groups. The first of them embeds most of the Nordic and continental welfare states. The second group is only made up with Luxembourg and Ireland, that are the countries with the lowest social spending but at the same time, they are within the group of efficient countries. The third group is made up of Mediterranean welfare states and United Kingdom and they are the less efficient countries in reducing vulnerability indexes.
\end{abstract}

Keywords: income inequality, poverty risk, efficient frontiers, data envelopment analysis, fuzzy clustering.

JEL classification: C49; F02; H50; H53.

MSC2010: 03E72; 62A86; 90C08.

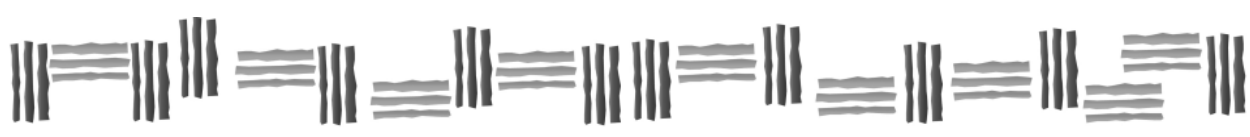




\section{Introducción.}

Un aspecto de interés en el análisis de sistemas de bienestar es la relación entre el volumen del gasto social (GS) y los indicadores de vulnerabilidad, como el riesgo de pobreza y el índice de desigualdad de rentas (índice Gini). Es necesario constatar la existencia de diferentes orientaciones en las políticas sociales según los países, dependiendo de las problemáticas sociales concretas, de las políticas sociales preexistentes, los sistemas de regulación del empleo y, más genéricamente, del estado del bienestar (EB). La Tabla 1 muestra que los países con mayor volumen de gasto social en relación al PIB son aquellos que presentan menor riesgo de pobreza después de prestaciones sociales. Son abundantes los estudios empíricos que han puesto de manifiesto la fuerte relación negativa entre el $G S$ y la pobreza y la desigualdad de ingresos, como por ejemplo en Cantillon et al. (1997), Bradbury y Jäntti (2001), Atkinson (2000), Beblo y Knaus (2001), Oxley et al. (2001).

Cantillon, Marx y Van den Bosch (2002) ponen de manifiesto que a pesar de la existencia de una fuerte correlación negativa entre gasto social y tasas de pobreza para diferentes países europeos, no se puede derivar un camino directo consistente en aumentar el gasto social para reducir la pobreza. Los autores realizan una simulación a partir de los datos del Luxembourg Income Study y sugieren que invertir más dinero en sistemas de transferencia social tendría resultados desiguales en la disminución del riesgo de pobreza en función del país. Si el gasto social fuera el único camino para reducir la pobreza, las recomendaciones políticas serían simples: aumentar el gasto (y/o mejorar su focalización en los países en los que ya es grande). La expansión del gasto no siempre tendría un fuerte efecto en la rebaja de las tasas de pobreza. La convergencia de los gastos sociales no produciría automáticamente una convergencia de las tasas de pobreza y este fenómeno sería más evidente en el caso de países como España e Italia, según la simulación realizada. Los autores sugieren que para el caso de España e Italia, las transferencias sociales aumentadas podrían ir a parar en forma de ingresos a las capas sociales intermedias y no pobres. Además, una distribución muy desigual de los salarios o un gran volumen de salarios precarios podrían hacer más difícil la redistribución de rentas. El efecto marginal de más gasto difiere considerablemente de un país a otro y no siempre es lineal.

Vandenbroucke y Vleminckx (2011) advierten que factores como la re-commodification o la resource competition en los nuevos Estados de bienestar, pueden cuestionar la relación existente entre gasto social y la lucha contra la pobreza, en la medida en que forman parte de la nueva configuración de las sociedades postindustriales y del papel que debe jugar el estado, las empresas y las entidades sociales como proveedores de bienestar. De manera similar, Cantillon (2011) cuestiona la estrategia de Lisboa argumentando que las transferencias monetarias dirigidas a personas sin trabajo no han tenido los efectos esperados y que las políticas redistributivas tampoco han sido suficientemente eficaces para paliar la pobreza. Varios estudios han manifestado el alcance limitado de las políticas de asistencia social, como Cincinnato y Nicaise (2009) para el caso de Bélgica, Bogdanov y Zahariev (2009), para Bulgaria, Anker et al. (2009) en Dinamarca, Ruoppila y Lamminmäki (2009) en Finlandia, Legros (2009) en Francia, Radu (2009) en Rumanía, Nelson (2003) en Suecia, Finn et al. (2008) en el Reino Unido.

Las consideraciones anteriores motivan el objetivo del presente trabajo, que evalúa la eficiencia del gasto social de los Estados que forman parte de la Unión Europea desde antes del 2000 (EU15) en la reducción de pobreza y la desigualdad de rentas durante el periodo 2011-2015. Tal como indican Afonso et al. (2010), existe una amplia literatura sobre eficiencia de políticas de gasto público, pero sobre todo en sanidad y enseñanza. Podemos remarcar Gupta y Verhoeven (2001), Clements (2002), Afonso y St Aubyn (2004, 2006), Herrera y Pang (2005), Agasisti y Johnes (2010) o Kapsoli y Teodoru (2017). Sin embargo, en nuestro caso buscamos determinar hasta qué punto los resultados alcanzados en reducción de la pobreza y desigualdad, que serán los outputs evaluados, se corresponde con el gasto social medido en relación al PIB y la situación inicial de la situación de vulnerabilidad analizada antes de gasto (inputs). Así, nuestro trabajo iría en la línea del trabajo de Afonso et al. (2010), elaborado para los países del ámbito de la OCDE y con datos del año 2000. 
En la EU15 se diferencian las siguientes tipologías de estado del bienestar: el modelo nórdico, (Dinamarca, Finlandia, Suecia y los Países Bajos), el modelo continental (Austria, Bélgica, Francia, Alemania y Luxemburgo), el modelo anglosajón (Irlanda y el Reino Unido) y el modelo mediterráneo (Grecia, Italia, Portugal y España). Con el objetivo de validar si la clasificación de los EB realmente se acomoda a la evidencia empírica referente al esfuerzo en gasto social y su efectividad en la disminución de los índices de vulnerabilidad realizamos un análisis clúster. En este análisis utilizaremos un enfoque de fuzzy clustering (Bezdek, 1981) en lugar de las metodologías de clustering más convencionales. Cuando usamos un algoritmo cluster k-means convencional todos los elementos analizados obligatoriamente se clasifican exclusivamente en un clúster. No obstante, en los fenómenos sociales la pertenencia exclusiva a una determinada tipología es normalmente irreal y simplificadora. Así, citando a Kandel (1982) la principal relación entre la teoría de los subconjuntos borrosos y los problemas de reconocimiento de patrones y clasificación recae en que en los problemas reales la mayor parte de categorías son difusas por naturaleza. Con fuzzy clustering podremos captar la posible ambigüedad de algunos estados al intentarlos clasificar en una única tipología, pero también reflejará la pertenencia inequívoca de otros a un grupo concreto.

El trabajo se ha estructurado de la siguiente manera: después de la introducción, se presentan los datos empleados en el análisis y se realiza un primer análisis descriptivo de sus relaciones. Posteriormente, la metodología utilizada para calcular la productividad del gasto social y la aplicación a los países EU15. A continuación, se realiza un análisis clúster que lleva a detectar las principales tipologías en el binomio gasto social/productividad en la reducción de los índices de vulnerabilidad. Finalmente, se presentan las principales conclusiones que se extraen del análisis.

\section{Base de datos y análisis descriptivo de las relaciones entre gasto social e indicadores de vulnerabilidad.}

El análisis se basa en datos de Eurostat relativos al periodo 2011 a 2015 (Tabla 1). El riesgo de pobreza antes de ejecutarse prestaciones sociales (a efectos de aligerar la lectura, se asimilan los conceptos de transferencias sociales y prestaciones sociales) y el índice de Gini antes de realizarse estas prestaciones presentan correlación positiva y significativa $\left(r_{x, y}=0,617\right)$. En lo que respecta al índice de Gini y el riesgo de pobreza después de prestaciones, la correlación es significativa y alta $\left(r_{x, y}=0,883\right)$ (Tabla 2$)$. El riesgo de pobreza antes de prestaciones $(R P a)$ y su valor después $(R P p)$ están altamente correlacionados $\left(r_{x, y}=0,831\right)$. Una correlación positiva, pero no tan alta, también la observamos entre el índice de Gini antes de prestaciones $(I G a)$ y después de estas $(I G p)\left(r_{x, y}=0,563\right)$. Tal como podíamos esperar, la correlación entre el nivel de $G S$ y $R P p$ e $I G p$ es negativa y significativa, al menos, con un nivel del 10\%: en ambos casos la correlación se sitúa en -0,527 y -0,466.

Tabla 1. Datos de partida para la evaluación de la eficiencia del gasto social en relación a la reducción de la pobreza en los países de la UE (15) referentes al periodo 2011-2015.

\begin{tabular}{|l|c|c|c|c|c|}
\hline & $\boldsymbol{G S}$ & $\boldsymbol{R P a}$ & $\boldsymbol{R P p}$ & $\boldsymbol{I G a}$ & IGp \\
\hline Francia & 33,64 & 44,34 & 13,66 & 50,1 & 29,66 \\
\hline Dinamarca & 32,34 & 40,84 & 12,02 & 51,62 & 27,22 \\
\hline Finlandia & 30,72 & 42,68 & 12,36 & 47,24 & 25,5 \\
\hline Holanda & 30,7 & 37,84 & 11,28 & 45,88 & 26,06 \\
\hline Bélgica & 29,98 & 43,02 & 15,26 & 47,84 & 26,16 \\
\hline Italia & 29,48 & 45,78 & 19,74 & 48,96 & 32,62 \\
\hline
\end{tabular}




\begin{tabular}{|l|c|c|c|c|c|} 
Austria & 29,46 & 44,26 & 14,18 & 47,36 & 27,32 \\
\hline Suecia & 29,24 & 44,54 & 15,86 & 56,62 & 26,64 \\
\hline Alemania & 28,88 & 43,66 & 16,42 & 56,08 & 29,66 \\
\hline Reino Unido & 28,44 & 44,04 & 16,24 & 54,68 & 31,4 \\
\hline Grecia & 26,8 & 52,24 & 22,18 & 60,18 & 34,34 \\
\hline Portugal & 26,48 & 46,8 & 18,92 & 59,36 & 34,22 \\
\hline España & 25,32 & 46,12 & 21,56 & 50,08 & 34,34 \\
\hline Luxemburgo & 22,42 & 45 & 15,84 & 48,44 & 29,32 \\
\hline Irlanda & 22,1 & 47,72 & 16,32 & 52,5 & 30,32 \\
\hline
\end{tabular}

Los países están ordenados según el Gasto social $(G S)$.

$R P p$ : Riesgo de pobreza después de prestaciones 2011-2015 (base 100)

$R P a$ : Riesgo de pobreza antes de prestaciones 2011-2015 (base 100)

GS: Gasto social como porcentaje del PIB 2011-2015

IGp: Índice de Gini después de prestaciones 2011-2015 (base 100)

IGa: Índice de Gini antes de prestaciones 2011-2015 (base 100)

Fuente: Elaboración propia a partir de datos de Eurostat, 2018.

Tabla 2. Correlaciones entre las diferentes variables empleadas en el estudio de la pobreza y su significación.

\begin{tabular}{|c|c|c|c|c|c|}
\hline & $\boldsymbol{R P p}$ & $\boldsymbol{R P a}$ & $\boldsymbol{I G} \boldsymbol{p}$ & $\boldsymbol{I G} \boldsymbol{a}$ & $\boldsymbol{G S}$ \\
\hline $\boldsymbol{R P} \boldsymbol{p}$ & 1 & & & & \\
\hline $\boldsymbol{R P a}$ & 0,8308 & & & & \\
\hline & $\left(4,611^{* * *}\right)$ & 1 & & & \\
\hline $\boldsymbol{G} \boldsymbol{p}$ & 0,8839 & 0,7414 & & & \\
$\boldsymbol{I} \boldsymbol{G} \boldsymbol{a}$ & $\left.0,396^{* * *}\right)$ & $\left(3,693^{* * *}\right)$ & 1 & & \\
\hline $\boldsymbol{G S}$ & $\left(2,3906^{* *}\right)$ & $\left(2,795^{* * *}\right)$ & $\left(2,468^{* *}\right)$ & 1 & \\
\hline
\end{tabular}

Nota: Entre paréntesis el valor del estadístico $z$ de prueba y con “***”, “**”, “*” se denota la significación estadística del coeficiente de correlación con un nivel de significación estadística del 1\%, 5\% y $10 \%$ respectivamente.

Fuente: Elaboración propia a partir de datos de Eurostat, 2018.

A partir de los datos de la Tabla 1 se miden los resultados conseguidos en políticas de reducción de los índices de vulnerabilidad en términos absolutos. La reducción absoluta del riesgo de pobreza $(R A P)$ se expresa:

$$
R A P=I P a-I P p
$$

De manera análoga se mide la reducción de la desigualdad en el reparto de la renta en términos absolutos $(R A G)$ :

$$
R A G=I G a-I G p
$$

La Tabla 3 recoge la posición (rango) que ocupa cada país de EU15 en cuanto a $R A P$ y a $R A G$. Aquí se evalúa únicamente el resultado final del $G S$ en la reducción de la pobreza y la desigualdad, sin ponderar el GS ni la situación de los indicadores antes de ejecutar el mismo. Los rangos correspondientes a $R A P$ y $R A G$ están correlacionados con baja intensidad; efectivamente, no generan jerarquías idénticas y, en cualquier caso, las correlaciones obtenidas (tanto de Pearson como de 
Spearman) no son significativas. Así, mientras que hay países que tienen un rango destacable en $R A P$ y uno más discreto en $R A G$ (Francia, Luxemburgo y Austria), otros, como Reino Unido, Alemania y Portugal presentan el comportamiento contrario. Finalmente, España o Italia tienen una jerarquía similar en ambos indicadores. Así pues, la correlación positiva pero no significativa de ambas variables, $R A P$ y $R A G$, ofrece la idea de cierta relación entre las magnitudes, pero en absoluto elevada.

Tabla 3. Jerarquía de países según la reducción absoluta del riesgo de pobreza y del índice de Gini $(R A P$ y $R A G)$ y rangos de la reducción, en el periodo 2011-2015.

\begin{tabular}{|l|c|c|c|c|}
\hline & $R A P$ & $R A G$ & Rango RAP & Rango RAG \\
\hline Alemania & 27,24 & 26,42 & 12 & 2 \\
\hline Austria & 30,08 & 20,04 & 4 & 9 \\
\hline Bélgica & 27,76 & 21,68 & 71 & 5 \\
\hline Dinamarca & 28,82 & 24,4 & 15 & 15 \\
\hline España & 24,56 & 15,74 & 3 & 8 \\
\hline Finlandia & 30,32 & 21,74 & 2 & 10 \\
\hline Francia & 30,68 & 20,44 & 5 & 12 \\
\hline Grecia & 30,06 & 25,84 & 13 & 7 \\
\hline Holanda & 26,56 & 19,82 & 1 & 14 \\
\hline Irlanda & 31,4 & 22,18 & 14 & 13 \\
\hline Italia & 26,04 & 16,34 & 6 & 4 \\
\hline Luxemburgo & 29,16 & 19,12 & 9 & 6 \\
\hline Portugal & 27,88 & 25,14 & 10 & 1 \\
\hline Reino Unido & 27,8 & 23,28 & 8 & \\
\hline Suecia & 28,68 & 29,98 & & \\
\hline
\end{tabular}

Nota: La correlación de Pearson entre $R A P$ y $R A G$ es 0,3397 y la de Spearman 0,2393 y en ambos casos no son significativas.

Fuente: Elaboración propia a partir de datos de Eurostat, 2018.

\section{Evaluación de la eficiencia del gasto social en la reducción de la pobreza y la desigualdad de rentas.}

\subsection{Obtención de fronteras productivas mediante el análisis envolvente de datos.}

El análisis de la eficiencia del gasto social se lleva a cabo con la técnica de Análisis Envolvente de Datos (DEA, Data Envelopment Analisis), propuesto en el clásico trabajo de Charnes et al. (1978). Esta metodología permite valorar la eficiencia de las políticas de los países de la EU15 respecto a la reducción de la desigualdad de rentas y la pobreza, tanto individualmente como considerando conjuntamente ambos objetivos. Se trata seguramente de la técnica analítica más ampliamente utilizada en análisis de productividad, como ponen de manifiesto Liu et al. (2013) y Emrounejad y Yang (2018). Es amplia literatura sobre la eficiencia de políticas públicas aplicando DEA, sobre todo en los ámbitos de sanidad, enseñanza e investigación. Al respecto, destacan los trabajos de Gupta y Verhoeven (2001), Clements (2002), Afonso y St. Aubyn (2004, 2006), González-Pérez et al. (2007), Coccia et al. (2015), Kapsoli y Teodoru (2017) y Anderson et al. (2017). En cambio, escasean los trabajos en el ámbito concreto que nos ocupa, la eficiencia del gasto social en la disminución de los indicadores de vulnerabilidad. Como trabajo más relevante se puede destacar Afonso et al. (2010), realizado con datos de principios del XXI del Luxemburg Income Study. En nuestro caso utilizamos datos más actualizados y nos centramos en los países de la EU15. 
El análisis DEA parte de que el conjunto de $n$ unidades decisoras (UD) evaluadas producen $R$ outputs a partir de $J$ inputs. Así, denotamos como $y_{r i}$ a la cantidad de output del tipo $r$ producido por la $i$-ésima unidad decisora, donde $i=1,2, \ldots, n, \mathrm{y} r=1,2, \ldots, R$ mientras que $x_{j i}$ es la cantidad de input $j$ utilizado por dicha UD siendo $j=1,2, \ldots, J$. El trabajo clásico de Charnes et al. (1978) asigna como ratio de eficiencia para la unidad productiva o-ésima $\theta_{O}$ al valor máximo del ratio de productividad, $\theta=\frac{\sum_{r=1}^{R} u_{r} y_{r o}}{\sum_{j=1}^{J} v_{r} x_{r o}}$. Así, para medir la productividad de la unidad productiva o-ésima debemos resolver el programa matemático:

$$
\max _{u_{r}, v_{j}} \theta=\frac{\sum_{r=1}^{R} u_{r} y_{r o}}{\sum_{j=1}^{J} v_{j} x_{j o}}
$$

sujeto a:

$$
\begin{gathered}
\frac{\sum_{r=1}^{R} u_{r} y_{r i}}{\sum_{j=1}^{J} v_{j} x_{j i}} \leq 1, i=1,2, \ldots, n \\
u_{r}>0, r=1,2, \ldots, R ; v_{j} \geq 0, j=1,2, \ldots, J
\end{gathered}
$$

Este programa matemático, a priori planteado como programa fraccional, puede transformarse en un programa lineal, sin más que suponer que $\sum_{j=1}^{J} v_{j} x_{r o}=1$ y transformar las $n$ restricciones del ratio de productividad de cada país por $\sum_{r=1}^{R} u_{r} y_{r i}-\sum_{j=1}^{J} v_{j} x_{r i} \leq 0, i=1,2, \ldots n$. Así, si en programa de maximización $\theta_{O}=1$, no podemos descartar que la unidad de decisión evaluada sea eficiente respecto al resto de la muestra mientras que si $\theta_{O}<1$ no hay ninguna duda de que la o-ésima unidad decisora es ineficiente. Según Sherman y Zhu (2006), el método DEA da a las UD el "beneficio de la duda" en el sentido en que el programa matemático (3) busca obtener los pesos $u_{r}$ y $v_{j}$ de tal manera que la unidad productiva evaluada sea presentado como lo más eficiente posible en comparación del resto de UDs. como:

Es más habitual resolver el programa (3) a partir del programa dual asociado a (3). Éste se plantea

$$
\min _{\theta, \lambda_{i}} \theta
$$

sujeto a:

$$
\begin{gathered}
\sum_{i=1}^{n} \lambda_{i} x_{j i} \leq \theta x_{j o}, j=1,2, \ldots, J \\
\sum_{i=1}^{n} \lambda_{i} y_{r i} \leq y_{r o}, r=1,2, \ldots, R \\
\sum_{i=1}^{n} \lambda_{i} \leq 1 \\
\lambda_{i} \geq 0, i=1,2, \ldots, n
\end{gathered}
$$

donde la introducción en el programa (4) por Banker et al. (1984) de la restricción $\sum_{i=1}^{n} \lambda_{i} \leq 1$ posibilita la consideración de que los rendimientos no sean constantes a escala. 


\subsection{Evaluación de la eficiencia del gasto social en la reducción absoluta del riesgo de pobreza $(R A P)$ y de la disminución de la desigualdad $(R A G)$.}

En primer lugar, se valora la eficiencia del gasto social de los países de la UE de forma individual en la reducción absoluta de la tasa de pobreza, $R A P$ y en la disminución absoluta de la desigualdad de rentas, $R A G$. Se considera en ambos casos como factores determinantes el gasto social $(G S)$ y el riesgo de pobreza y el índice de Gini antes de prestaciones. Posteriormente, se valora el logro conjunto de ambos objetivos. Se aplica en todos los casos la metodología DEA descrita, con el programa dual (4). Así, para evaluar la eficiencia del gasto social del o-ésimo estado en la reducción de la pobreza exclusivamente planteamos:

$$
\min _{\theta, \lambda_{i}} \theta
$$

sujeto a:

$\sum_{i=1}^{15} \lambda_{i} G S_{i} \leq \theta G S_{o} ; \sum_{i=1}^{15} \lambda_{i} R P a_{i} \leq \theta R P a_{o} ; \sum_{i=1}^{15} \lambda_{i} R A P_{i} \leq R A P_{o} ; \sum_{i=1}^{15} \lambda_{i} \leq 1 ; \lambda_{i} \geq 0, i=1,2, \ldots, 15$

(5)

Para determinar la eficiencia en la disminución de la desigualdad de rentas basta con plantear un programa análogo a (5) donde $R P a_{i}$ quedará sustituido por $I G a_{i}$ y $R A P_{i}$ debe reemplazarse por $R A G_{i}$. Concretamente plantearíamos:

$$
\min _{\theta, \lambda_{i}} \theta
$$

sujeto a:

$\sum_{i=1}^{15} \lambda_{i} G S_{i} \leq \theta G S_{o} ; \sum_{i=1}^{15} \lambda_{i} R G a_{i} \leq \theta R G a_{o} ; \sum_{i=1}^{15} \lambda_{i} R A G_{i} \leq R A G_{o} ; \sum_{i=1}^{15} \lambda_{i} \leq 1 ; \lambda_{i} \geq 0, i=1,2, \ldots, 15$

La utilización de los métodos DEA permite la evaluación conjunta de $R A P$ y $R A G$. Para ello planteamos un programa matemático que combine (5) y (6) de la siguiente forma:

$$
\min _{\theta, \lambda_{i}} \theta
$$

sujeto a:

$$
\begin{aligned}
& \sum_{i=1}^{15} \lambda_{i} G S_{i} \leq \theta G S_{o} ; \sum_{i=1}^{15} \lambda_{i} R P a_{i} \leq \theta R P a_{o} ; \sum_{i=1}^{15} \lambda_{i} I G a_{i} \leq \theta I G a_{o} ; \sum_{i=1}^{15} \lambda_{i} R A P_{i} \leq R A P_{o} \\
& \sum_{i=1}^{15} \lambda_{i} R A G_{i} \leq R A G_{o} ; \sum_{i=1}^{15} \lambda_{i} \leq 1 ; \lambda_{i} \geq 0, i=1,2, \ldots, 15
\end{aligned}
$$

En la Tabla 4 se muestran los valores y los rankings obtenidos de la resolución de los modelos (5), (6) y (7). Teniendo en cuenta, exclusivamente, la eficiencia en la reducción de la pobreza, se observa que los países menos eficientes son en general los que tienen un estado del bienestar de tipo mediterráneo (Grecia, Italia y Portugal), mientras que los más eficientes pueden pertenecer tanto al modelo tanto nórdico (Finlandia y Holanda) como anglosajón (Irlanda) o continental (Francia). 
Tabla 4. Medidas de eficiencia de DEA de los países de la EU15.

\begin{tabular}{|c|c|c|c|c|c|c|}
\hline & $\begin{array}{l}\text { Eficiencia } \\
\text { según }(5)\end{array}$ & $\begin{array}{l}\text { Eficiencia } \\
\text { según (6) }\end{array}$ & $\begin{array}{l}\text { Eficiencia } \\
\text { según (7) }\end{array}$ & $\begin{array}{l}\text { Jerarquía } \\
\text { según (5) }\end{array}$ & $\begin{array}{l}\text { Jerarquía } \\
\text { según (6) }\end{array}$ & $\begin{array}{l}\text { Jerarquía } \\
\text { según (7) }\end{array}$ \\
\hline Alemania & 0,9381 & 0,9565 & 0,9903 & 12 & 9 & 10 \\
\hline Austria & 0,9761 & 0,9851 & 1 & 7 & 7 & 1 \\
\hline Bélgica & 0,9408 & 0,995 & 0,9953 & 10 & 6 & 9 \\
\hline Dinamarca & 0,9978 & 0,9738 & 1 & 6 & 8 & 1 \\
\hline España & 0,9467 & 0,9562 & 0,9562 & 8 & 10 & 13 \\
\hline Finlandia & 1 & 1 & 1 & 1 & 1 & 1 \\
\hline Francia & 1 & 0,9245 & 1 & 1 & 15 & 1 \\
\hline Grecia & 0,8719 & 0,9496 & 0,9496 & 15 & 12 & 15 \\
\hline Holanda & 1 & 1 & 1 & 1 & 1 & 1 \\
\hline Irlanda & 1 & 1 & 1 & 1 & 1 & 1 \\
\hline Italia & 0,9034 & 0,9535 & 0,9535 & 14 & 11 & 14 \\
\hline Luxemburgo & 1 & 1 & 1 & 1 & 1 & 1 \\
\hline Portugal & 0,9238 & 0,9369 & 0,97 & 13 & 13 & 11 \\
\hline Reino Unido & 0,9381 & 0,9307 & 0,9669 & 11 & 14 & 12 \\
\hline Suecia & 0,9412 & 1 & 1 & 9 & 1 & 1 \\
\hline
\end{tabular}

Fuente: Elaboración propia a partir de datos de Eurostat, 2018.

En cuanto a la eficiencia exclusiva en la reducción de $I G$, (5), se observa una tónica similar, pero con matices. Entre los países más ineficientes encontramos de nuevo los países mediterráneos y también el Reino Unido (éste estaba jerarquizado en posiciones retrasadas al evaluarse exclusivamente la eficiencia en $R A P$ ) y, sorprendentemente, Francia, que estaba entre los países más eficientes en $R A P$. En cuanto a los países más eficientes, países con EB de modelo nórdico (Finlandia, Holanda, Suecia) y también los dos países que presentan menor gasto social, Luxemburgo e Irlanda. En general los países con EB continental se sitúan en posiciones intermedias.

Cuando se evalúa conjuntamente la eficiencia en $R A P$ y $R A G$ (modelo (7)), los países eficientes vuelven a ser, esencialmente, países con modelo de EB nórdico (Holanda, Dinamarca, Suecia y Finlandia), más Francia y países con menor gasto social (Luxemburgo e Irlanda). Los países más ineficientes son los mediterráneos y el Reino Unido, nuevamente. En las posiciones centrales encontramos, esencialmente, países con EB continentales.

En la Tabla 5 podemos observar que la eficiencia en $R A P$ y $R A G$ está positivamente relacionada (Rho de Spearman $=0,583$ ), y aun siendo estadísticamente significativa, está lejos de ser perfecta. Hay países eficientes en $R A P$ e ineficientes en $R A G$ (Francia) y viceversa (Suecia).

Tabla 5. Correlación por rangos de Spearman de las medidas de eficiencia de los modelos (5), (6) y (7).

\begin{tabular}{|c|c|c|c|}
\hline & Jerarquía en (5) & Jerarquía en (6) & Jerarquía en (7) \\
\hline Jerarquía en (5) & 1 & & \\
\hline Jerarquía en (6) & 0,5826 & 1 & \\
\hline Jerarquía en (7) & $\left(2,581^{* *}\right)$ & 0,6514 & 1 \\
\hline
\end{tabular}

Nota: Entre paréntesis el valor del estadístico $z$ de prueba y con “***”, “**”, “*” se denota significación estadística del coeficiente de correlación con un nivel de significación estadística del 1\%, 5\% y 10\% respectivamente. Fuente: Elaboración propia a partir de datos de Eurostat, 2018. 


\section{Detección de patrones en las políticas públicas de pobreza y desigualdad en los países de la EU15.}

\subsection{Análisis clúster $k$-means borroso versus $\boldsymbol{k}$-means convencional.}

El análisis clúster es un método utilizado para la detección de grupos homogéneos dentro de una muestra de estudio. Los algoritmos clúster $k$-means convencionales permiten clasificar cada elemento de un conjunto de $n$ elementos exclusivamente en un grupo de los $K$ preestablecidos, considerándose la exclusividad de pertenencia. Una vez se ha fijado el número de clústeres deseados, la determinación de centroides busca minimizar la dispersión de los elementos dentro de un grupo como:

$$
\begin{array}{r}
\underset{c_{k}, u_{i, k}}{\operatorname{Min}} \sum_{k=1}^{K} \sum_{i=1}^{n} u_{i, k}\left\|x_{i}-c_{k}\right\|^{2} \\
\text { sujeto a } \sum_{k=1}^{K} u_{i, k}=1, u_{i, k} \in\{0,1\}
\end{array}
$$

Siendo $\|\cdot\|$ una norma normalmente euclídea, $x_{i}$ la observación del $i$-ésimo elemento sobre un conjunto de características, $c_{k}$ el prototipo o centroide del clúster $k$ y $u_{i, k}$ el nivel de pertenencia del elemento $i$ al clúster $k, u_{i, k} \in\{0,1\}$. Así, $u_{i, k}=1$ si el elemento $i$-ésimo es clasificado en el grupo $k$ y $u_{i, k}$ $=0$ en caso contrario.

La modelización fuzzy k-means relaja la condición de exclusividad de pertenencia a un grupo y supone que cualquier elemento puede pertenecer en un cierto grado delimitado en el intervalo [0,1], a más de uno de los grupos prefijados. Así, el programa de optimización anterior queda re-expresado como (8b):

$$
\begin{aligned}
& \underset{c_{k}, u_{i, k}}{\operatorname{Min}} J=\sum_{k=1}^{K} \sum_{i=1}^{n}\left(u_{i, k}\right)^{m}\left\|x_{i}-c_{k}\right\|^{2} \\
& \text { sujeto a } \sum_{k=1}^{K} u_{i, k}=1,0 \leq u_{i, k} \leq 1
\end{aligned}
$$

donde el parámetro $m$ es denominado en la literatura como fuzzificador y puede tomar valores 1 $\leq m<\infty$. Cuando $m \rightarrow 1$, la partición resultante es una convencional (nítida) mientras que si $m \rightarrow \infty$ el análisis clúster no añade valor ya que $u_{i, k} \rightarrow \frac{1}{K}$. Tal como indican Klawonn et al. (2015), el análisis clúster convencional puede ser entendido como un caso particular del análisis clúster borroso ya que los grupos borrosos pueden ser transformados en grupos nítidos considerando que cualquier observación $x_{i}$ pertenece exclusivamente al clúster $s$ si $u_{i, s}=\max _{k=1}^{K} u_{i, k}$.

En problemas de clasificación relativos a las Ciencias Sociales, la definición de las clases suele ser difusa, de tal forma que muchos de los elementos a clasificar pueden participar de características de más de un grupo. Así, si se establecen dos grupos en función de la eficiencia en $R A P$ "alta eficiencia en RAP" y "baja eficiencia en RAP", la misma naturaleza con la que hemos etiquetado estas clases económicas nos lleva a considerar el fuzzy clustering como una alternativa interesante al hard clustering. Así, aceptaríamos que la pertenencia de Luxemburgo al primer grupo y Grecia el segundo es inequívoca, pero Dinamarca, con un índice de eficiencia en $R A P$ de 0,99 , muy cercano a 1 , participaría, en cierto grado, de ambos grupos. El análisis fuzzy clustering ha sido ampliamente utilizado en problemas de clasificación de tipo económico y social. Así, Derrig y Ostaszewski (1995) clasifican varios municipios del estado de Massachussets en función de variables relacionadas con el fraude en seguros de automóvil; Yu et al. (2012) y Yu et al. (2014) clasifican las provincias chinas en función del cumplimiento de los objetivos de emisiones en $\mathrm{CO}^{2}$ y Wu et al. (2013) categorizan las regiones chinas en función de la vulnerabilidad de su producción agrícola. En Khoshnevisan et al. (2015) se clasifican explotaciones agrarias de Irán en función de su eficiencia energética. En el ámbito 
de la gestión empresarial, Costea y Bleotu (2012) aplica el fuzzy clustering para discriminar instituciones financieras en Rumania, mientras que Khaleie et al. (2012) lo hace en la selección de proveedores y Hirschinger et al. (2015) en problemas de logística.

Klawonn et al. (2015) introducen una nueva razón de tipo algorítmico para emplear métodos de fuzzy clustering. Indican que el clustering borroso en muchas ocasiones evita los problemas algorítmicos de los métodos convencionales clúster k-means como, por ejemplo, que sus resultados dependen fuertemente de la inicialización del algoritmo. Además, fuzzy clustering no es únicamente una simple mejora de los algoritmos convencional k-clustering sino que abre la posibilidad de realizar análisis clúster más flexibles y sofisticados que los que se pueden hacer con el análisis clúster nítido. La obtención de los centroides y los niveles de pertenencia asociados a los grupos puede ser resuelto con el algoritmo propuesto en Bezdek (1981). Éste se basa en la aplicación recurrente de las siguientes ecuaciones. La primera estipula que el centroides del clúster k-ésimo es la media ponderada:

$$
c_{k}=\frac{\sum_{i=1}^{n}\left(u_{i, k}\right)^{m} x_{i}}{\sum_{i=1}^{n}\left(u_{i, k}\right)^{m}}
$$

La segunda ecuación estipula que el nivel de pertenencia del elemento $i$-ésimo al k-ésimo clúster, $u_{i, k}$, se encuentra como:

$$
u_{i, k}=\left[\sum_{s=1}^{K}\left(\frac{\left\|x_{i}-c_{k}\right\|^{2}}{\left\|x_{i}-c_{s}\right\|^{2}}\right)^{\frac{1}{m-1}}\right]^{-1}
$$

Así, los pasos que sigue el algoritmo de Bezdeck son los siguientes:

Paso 1: A partir de un número predefinido del número de clústeres, $K$ y de $m$, escoge un nivel inicial de pertenencia de cada elemento a cada clúster $u_{i, k}^{(0)}$, de tal manera que $\sum_{k=1}^{K} u_{i, k}^{(0)}=1$.

Paso 2: Con (8c) calcula el centroide del clúster k-ésimo de la primera iteración, $c_{k}^{(0)}, k=1,2, \ldots, K$.

Paso 3: Con (8d) calcula el nivel de pertenencia de cada elemento a cada clúster. En esta primera iteración obtenemos $u_{i, k}^{(1)} i=1,2, \ldots, n ; k=1,2, \ldots, K$.

Paso 4: Calcula el valor de la función de coste en $(8 \mathrm{~b}) \operatorname{con} u_{i, k}^{(1)}$. Si se produce en su valor una mejora respecto al asociado a $u_{i, k}^{(0)}$ en una cuantía superior a un valor predefinido $\varepsilon$, repite los tres pasos anteriores a partir del valor $u_{i, k}^{(1)}$ para encontrar $u_{i, k}^{(2)}$ e implementa este paso. Así, en la iteración $i$-ésima, si a partir de $u_{i, k}^{(i)}$ se produce una mejora en la función objetiva respecto a $u_{i, k}^{(i-1)}$ superior al valor predefinido $\varepsilon$, se vuelven a implementar los cuatro pasos descritos. El algoritmo finaliza en el momento en que la iteración no proporciona una mejora en la función de coste superior a $\varepsilon$.

Una cuestión relevante en el análisis fuzzy k-means es la elección del número óptimo de clústeres $K$. Esto ha generado una extensa literatura tal como se muestra en el survey de Wang y Zhang (2007). Aquí utilizamos dos índices de validación del número de clústeres que parten de una filosofía diferente. El primero únicamente utiliza los niveles de pertenencia y es un refinamiento que Dave (1996) hace sobre el clásico índice de Bezdek (1981). Denominamos este índice como IDB y lo calculamos como

$$
I D B=1-\frac{K}{K-1}\left(1-\frac{1}{n} \sum_{k=1}^{K} \sum_{i=1}^{n}\left(u_{i, k}\right)^{2}\right)
$$


El número óptimo de clústeres es aquel valor de que $K$ maximice $I D B$ y, básicamente, será aquella partición que se acerca a una partición hard.

El segundo índice que empleamos es el propuesto en Xie y Beni (1991), que Wang y Zhang (2007) entienden como más completo ya que, aparte de los niveles de pertenencia, utiliza el valor de las observaciones. El valor óptimo de $K$ debe minimizar la dispersión dentro de las clases y, al mismo tiempo, maximizar la dispersión entre clases. Si denominamos este índice como $I X B$, el $K$ óptimo deberá minimizarlo, ya que:

$$
I X B=\frac{\sum_{k=1}^{K} \sum_{i=1}^{n}\left(u_{i, k}\right)^{m}\left\|x_{i}-c_{k}\right\|^{2}}{n \cdot \min _{j, k}\left\|c_{j}-c_{k}\right\|^{2}}
$$

\subsection{Patrones en la eficiencia del gasto social de los estados de la EU15.}

Con la metodología fuzzy clustering ya descrita buscamos identificar los patrones existentes en los países EU15 en el volumen de políticas sociales y su productividad, medida a partir de la modelización DEA en tres niveles:

a) En primer lugar agrupamos los países por su volumen de $G S$ y su productividad en $R A P$. Este análisis será denotado como GS-RAP.

b) En segundo lugar repetimos el mismo análisis considerando la productividad de $G S$ en $R A G$. Nos referimos a este análisis como GS-RAG.

c) Finalmente teniendo en cuenta el volumen de $G S$ y su productividad tanto en $R A P$ como en $R A G$ $(G S-R A P / R A G)$.

En todas las variables, $G S$ y el índice de eficiencia, utilizamos sus valores estandarizados. Para establecer el número final de clústeres, tal como sugieren Yu et al. (2012), partiremos de un número máximo de $\sqrt{ } n=\sqrt{ } 15 \approx 4$ y un mínimo de 2 . Además, empleamos $m=2$, que suele ser la alternativa más común en trabajos empíricos. Calculamos los centroides de los clústeres y los niveles de pertenencia de los elementos de la muestra para $k=2,3$, y 4 y con los índices $I B X$ e $I D B$, establecemos la partición que entendemos óptima. En la Tabla 6 se recogen los valores que toman los índices $I D B$ y $I X B$ en los análisis clúster planteados. Puede observarse que en todos los casos los índices de validación sugieren que el número óptimo de grupos es $K=3$.

Tabla 6. Índices de validación de número de clústeres en un análisis fuzzy k-means.

\begin{tabular}{|c|c|c|c|c|c|c|}
\hline & \multicolumn{2}{|c|}{ Análisis GS-RAP } & \multicolumn{2}{c|}{ Análisis GS-RAG } & \multicolumn{2}{c|}{ Análisis GS-RAP/RAG } \\
\hline$k$ & $I D B$ & $I X B$ & $I D B$ & $I X B$ & $I D B$ & $I X B$ \\
\hline 2 & $-0,1043$ & 0,0850 & $-0,1714$ & 0,1365 & $-0,1583$ & 0,1521 \\
\hline 3 & $\mathbf{0 , 1 8 9 7}$ & $\mathbf{0 , 0 2 3 9}$ & $\mathbf{0 , 1 4 5 4}$ & $\mathbf{0 , 0 4 3 3}$ & $\mathbf{0 , 1 4 5 4}$ & $\mathbf{0 , 0 5 3 0}$ \\
\hline 4 & 0,1031 & 0,0995 & 0,1110 & 0,0657 & 0,0819 & 0,1455 \\
\hline
\end{tabular}

Fuente: Elaboración propia a partir de datos de Eurostat, 2018. 


\subsubsection{Patrones de eficiencia del gasto social en la reducción de la pobreza.}

La Tabla 7 recoge los valores estandarizados de GS y el índice de eficiencia y los niveles de pertenencia de cada país a los tres clústeres, mientras que la Tabla 8 muestra sus centroides.

De los tres grupos identificados, los dos primeros se componen de países que, en general, son eficientes en la reducción de la pobreza pero que difieren en el volumen del $G S$. El primer clúster, engloba países con EB nórdico (Dinamarca, Holanda y Finlandia) o continental (Austria y Francia). Estos países manifiestan eficiencia en la reducción de la pobreza y presentan un nivel de $G S$ claramente por encima del promedio de la EU15. En el segundo hay dos países, Luxemburgo e Irlanda, eficientes según el análisis DEA pero con $G S$ notablemente por debajo del promedio de la EU15. El sensible nivel de pertenencia a este segundo grupo de España se explica por su similitud en cuanto al GS (de los más reducidos de la EU15) y no por su grado de eficiencia, que está por debajo del promedio.

Tabla 7. Grupos del análisis cluster fuzzy k-means GS-RAP.

\begin{tabular}{|l|c|c|c|c|c|}
\hline & GS & Eficiencia en RAP & $\boldsymbol{u}_{\boldsymbol{i} 1}$ & $\boldsymbol{u}_{\boldsymbol{i} \mathbf{2}}$ & $\boldsymbol{u}_{\boldsymbol{i 3}}$ \\
\hline Alemania & 0,146 & $-0,496$ & 0,036 & 0,016 & $\mathbf{0 , 9 4 8}$ \\
\hline Austria & 0,323 & 0,426 & $\mathbf{0 , 6 5 9}$ & 0,082 & 0,258 \\
\hline Bélgica & 0,482 & $-0,43$ & 0,147 & 0,041 & $\mathbf{0 , 8 1 2}$ \\
\hline Dinamarca & 1,201 & 0,953 & $\mathbf{0 , 9 7 6}$ & 0,008 & 0,016 \\
\hline España & $-0,939$ & $-0,287$ & 0,128 & 0,286 & $\mathbf{0 , 5 8 5}$ \\
\hline Finlandia & 0,707 & 1,006 & $\mathbf{0 , 9 7 3}$ & 0,01 & 0,017 \\
\hline Francia & 1,598 & 1,006 & $\mathbf{0 , 8 9 5}$ & 0,035 & 0,07 \\
\hline Grecia & $-0,488$ & $-2,101$ & 0,134 & 0,136 & $\mathbf{0 , 7 3 1}$ \\
\hline Holanda & 0,701 & 1,006 & $\mathbf{0 , 9 7 2}$ & 0,01 & 0,018 \\
\hline Irlanda & $-1,921$ & 1,006 & 0,002 & $\mathbf{0 , 9 9 4}$ & 0,003 \\
\hline Italia & 0,329 & $-1,337$ & 0,074 & 0,041 & $\mathbf{0 , 8 8 5}$ \\
\hline Luxemburgo & $-1,824$ & 1,006 & 0,001 & $\mathbf{0 , 9 9 8}$ & 0,001 \\
\hline Portugal & $-0,585$ & $-0,842$ & 0,055 & 0,064 & $\mathbf{0 , 8 8 2}$ \\
\hline Reino Unido & 0,012 & $-0,496$ & 0,025 & 0,013 & $\mathbf{0 , 9 6 2}$ \\
\hline Suecia & 0,256 & $-0,42$ & 0,077 & 0,028 & $\mathbf{0 , 8 9 4}$ \\
\hline
\end{tabular}

Notas: (a) Los valores de $G S$ y la medida de eficiencia vienen estandarizados.

(b) La media y la desviación estándar de $G S$ son 28,400 y 3,279 respectivamente. Las del índice de eficiencia en $R A P$ son 0,956 y 0,041 .

Fuente: Elaboración propia a partir de datos de Eurostat, 2018.

En el tercer grupo, el volumen de $G S$ es muy heterogéneo aunque su promedio es cercano al promedio de la EU15. Agrupa a países menos eficientes en la reducción de la pobreza, comparativamente a los dos anteriores grupos. Están los países mediterráneos (Italia, España, Italia y Grecia), dos países con modelo EB continental (Alemania, Bélgica), que pasan a ser tres si consideramos a Austria (presenta un nivel de pertenencia al grupo de 0,258); y, finalmente, Suecia y Reino Unido. 
Tabla 8. Centroides de los clústeres identificados en la Tabla 7.

\begin{tabular}{|c|c|c|}
\hline & GS & Eficiencia en $\boldsymbol{R A P}$ \\
\hline Centroide del clúster 1 & 0,935 & 0,900 \\
\hline Centroide del clúster 2 & $-1,807$ & 0,920 \\
\hline Centroide del clúster 3 & $-0,015$ & $-0,762$ \\
\hline
\end{tabular}

Notas: (a) Los valores de $G S$ y la medida de eficiencia vienen estandarizados.

(b) La media y la desviación estándar de $G S$ son 28,400 y 3,279 respectivamente. Las del índice de eficiencia en $R A P$ son 0,956 y 0,041 .

Fuente: Elaboración propia a partir de datos de Eurostat, 2018.

\subsubsection{Patrones de eficiencia del gasto social en la reducción de la desigualdad}

La Tabla 9 recoge los valores estandarizados de $G S$ y de eficiencia en $R A G$ y los niveles de pertenencia de cada país a los tres clústeres identificados, mientras que en la Tabla 10 se indican sus centroides. Diferenciamos tres grupos, que presentan una configuración muy similar a los que detectamos en el análisis GS-RAP. Los dos primeros se componen de países que en general son eficientes en la disminución de la desigualdad pero que tienen un volumen muy diferente en gasto social. El primer grupo engloba países con EB nórdico (Dinamarca, Suecia, Holanda y Finlandia) y dos continentales (Austria y Bélgica). Francia presenta una sensible pertenencia a este grupo, más por su alto valor en gasto social que por su nivel de eficiencia al reducir la desigualdad. El segundo grupo se compone esencialmente de dos países (Luxemburgo e Irlanda) que según el análisis DEA son completamente eficientes pero con $G S$ notablemente por debajo del denominador común de la EU15. Llama de nuevo la atención la sensible pertenencia de España a este clúster, que puede imputarse a su patrón en $G S$ y no por su eficiencia en la disminución efectiva en $R A G$.

En el tercer grupo el $G S$ es muy heterogéneo, con promedio cerca del de la EU15. En este grupo todos los países son escasamente eficientes en la reducción de la desigualdad en comparación a los grupos anteriores. Se engloban aquí, de nuevo, los países mediterráneos (Italia, España, Italia y Grecia), dos países con EB continental (Alemania y Francia) y Reino Unido. También es remarcable la sensible pertenencia de Dinamarca, debido a que su eficiencia en $R A G(0,97)$, está sensiblemente alejada de la eficiencia plena (1).

Tabla 9. Grupos del análisis cluster fuzzy k-means GS-RAG.

\begin{tabular}{|l|c|c|c|c|c|}
\hline & $\boldsymbol{G S}$ & $\begin{array}{c}\text { Eficiencia en en } \\
\boldsymbol{R} \boldsymbol{A G}\end{array}$ & $\boldsymbol{u}_{\boldsymbol{i} \text { ( }}$ & $\boldsymbol{u}_{i 2}$ & $\boldsymbol{u}_{i 3}$ \\
\hline Alemania & 0,146 & $-0,507$ & 0,101 & 0,03 & $\mathbf{0 , 8 6 9}$ \\
\hline Austria & 0,323 & 0,508 & $\mathbf{0 , 9 1 9}$ & 0,025 & 0,055 \\
\hline Bélgica & 0,482 & 0,859 & $\mathbf{0 , 9 9 1}$ & 0,003 & 0,005 \\
\hline Dinamarca & 1,201 & 0,107 & $\mathbf{0 , 7 1 6}$ & 0,059 & 0,225 \\
\hline España & $-0,939$ & $-0,517$ & 0,156 & 0,204 & $\mathbf{0 , 6 4}$ \\
\hline Finlandia & 0,707 & 1,036 & $\mathbf{0 , 9 6 7}$ & 0,014 & 0,02 \\
\hline Francia & 1,598 & $-1,642$ & 0,286 & 0,105 & $\mathbf{0 , 6 0 8}$ \\
\hline Grecia & $-0,488$ & $-0,751$ & 0,059 & 0,042 & $\mathbf{0 , 8 9 8}$ \\
\hline Holanda & 0,701 & 1,036 & $\mathbf{0 , 9 6 7}$ & 0,013 & 0,019 \\
\hline Irlanda & $-1,921$ & 1,036 & 0,002 & $\mathbf{0 , 9 9 6}$ & 0,002 \\
\hline Italia & 0,329 & $-0,613$ & 0,104 & 0,028 & $\mathbf{0 , 8 6 7}$ \\
\hline
\end{tabular}




\begin{tabular}{|l|c|c|c|c|c|} 
Luxemburgo & $-1,824$ & 1,036 & 0,001 & $\mathbf{0 , 9 9 9}$ & 0 \\
\hline Portugal & $-0,585$ & $-1,202$ & 0,063 & 0,052 & $\mathbf{0 , 8 8 5}$ \\
\hline Reino Unido & 0,012 & $-1,422$ & 0,046 & 0,026 & $\mathbf{0 , 9 2 8}$ \\
\hline Suecia & 0,256 & 1,036 & $\mathbf{0 , 9 2 1}$ & 0,038 & 0,042 \\
\hline
\end{tabular}

Notas: (a) Los valores de $G S$ y la medida de eficiencia vienen estandarizados.

(b) La media y la desviación estándar de $G S$ son 28,400 y 3,279 respectivamente. Las del índice de eficiencia en $R A G$ son 0,971 y 0,028 .

Fuente: Elaboración propia a partir de datos de Eurostat, 2018.

Tabla 10. Centroides de los clústeres identificados en la Tabla 9.

\begin{tabular}{|c|c|c|}
\hline & $G S$ & Eficiencia en $R A G$ \\
\hline Centroide del clúster 1 & 0,935 & 0,900 \\
\hline Centroide del clúster 2 & $-1,807$ & 0,920 \\
\hline Centroide del clúster 3 & $-0,015$ & $-0,762$ \\
\hline
\end{tabular}

Notas: (a) Los valores de $G S$ y la medida de eficiencia vienen estandarizados.

(b) La media y la desviación estándar de $G S$ son 28,400 y 3,279 respectivamente. Las del índice de eficiencia en $R A G$ son 0,971 y 0,028 .

Fuente: Elaboración propia a partir de datos de Eurostat, 2018.

4.2.3. Patrones de eficiencia del GS en la reducción de la pobreza y la desigualdad considerados conjuntamente

La Tabla 11 recoge los valores estandarizados de $G S$ y de eficiencia conjunta en $R A P$ y $R A G$ y los niveles de pertenencia de cada país a los tres clústeres. En la Tabla 12 se indican sus centroides. Vuelven a diferenciarse tres grupos, no obstante, los grupos se muestran de forma mucho más nítida, ya que no hay países que pertenezcan a más de un grupo con niveles de presunción superiores a 0,1 . Los dos primeros clústeres se componen de países que son eficientes en la ejecución del $G S$ pero con un volumen muy diferente en el mismo. El primer grupo abarca todos los países con EB nórdico (Dinamarca, Suecia, Holanda y Finlandia) y continental (Austria, Bélgica, Francia y Alemania). El segundo grupo se conforma esencialmente de Luxemburgo e Irlanda, que se manifiestan eficientes según el análisis DEA, pero tienen un $G S$ notablemente por debajo del resto de países de la EU15. En el tercer grupo el $G S$ es muy heterogéneo y su promedio está por debajo, en cualquier caso, del de EB nórdico y continental. Se trata de países mediterráneos (Italia, España, Italia y Grecia), con el añadido del Reino Unido. 
Tabla 11. Grupos del análisis cluster fuzzy k-means GS-RAP y RAG.

\begin{tabular}{|l|c|c|c|c|c|}
\hline & $\boldsymbol{G S}$ & $\begin{array}{c}\text { Eficiencia } \\
\text { conjunta en en } \\
\text { RAP y RAG }\end{array}$ & $\boldsymbol{u}_{\boldsymbol{i} \text { i }}$ & $\boldsymbol{u}_{\text {i2 }}$ & $\boldsymbol{u}_{\boldsymbol{i 3}}$ \\
\hline Alemania & 0,146 & 0,245 & $\mathbf{0 , 7 8 8}$ & 0,083 & 0,129 \\
\hline Austria & 0,323 & 0,73 & $\mathbf{0 , 9 4 7}$ & 0,026 & 0,026 \\
\hline Bélgica & 0,482 & 0,496 & $\mathbf{0 , 9 7 5}$ & 0,011 & 0,015 \\
\hline Dinamarca & 1,201 & 0,73 & $\mathbf{0 , 9 3 1}$ & 0,029 & 0,04 \\
\hline España & $-0,939$ & $-1,469$ & 0,048 & 0,061 & $\mathbf{0 , 8 9}$ \\
\hline Finlandia & 0,707 & 0,73 & $\mathbf{0 , 9 9 7}$ & 0,001 & 0,001 \\
\hline Francia & 1,598 & 0,73 & $\mathbf{0 , 8 4 7}$ & 0,062 & 0,092 \\
\hline Grecia & $-0,488$ & $-1,798$ & 0,032 & 0,028 & $\mathbf{0 , 9 4}$ \\
\hline Holanda & 0,701 & 0,73 & $\mathbf{0 , 9 9 7}$ & 0,001 & 0,001 \\
\hline Irlanda & $-1,921$ & 0,73 & 0,001 & $\mathbf{0 , 9 9 8}$ & 0,001 \\
\hline Italia & 0,329 & $-1,606$ & 0,087 & 0,045 & $\mathbf{0 , 8 6 8}$ \\
\hline Luxemburgo & $-1,824$ & 0,73 & 0 & $\mathbf{1}$ & 0 \\
\hline Portugal & $-0,585$ & $-0,774$ & 0,085 & 0,08 & $\mathbf{0 , 8 3 5}$ \\
\hline Reino Unido & 0,012 & $-0,932$ & 0,082 & 0,039 & $\mathbf{0 , 8 7 8}$ \\
\hline Suecia & 0,256 & 0,73 & $\mathbf{0 , 9 2 5}$ & 0,038 & 0,037 \\
\hline
\end{tabular}

Notas: (a) Los valores de $G S$ y la medida de eficiencia vienen estandarizados.

(b) La media y la desviación estándar de $G S$ son 28,400 y 3,279 respectivamente. Las del índice de eficiencia en $R A P$ y $R A G$ conjuntamente son 0,985 y 0,02 .

Fuente: Elaboración propia a partir de datos de Eurostat, 2018.

Tabla 12. Centroides de los clústers identificados en la Tabla 11.

\begin{tabular}{|c|c|c|}
\hline & GS & Eficiencia en $\boldsymbol{R A P}$ y $\boldsymbol{R A G}$ \\
\hline Centroide del clúster 1 & 0,674 & 0,648 \\
\hline Centroide del clúster 2 & $-1,843$ & 0,714 \\
\hline Centroide del clúster 3 & $-0,336$ & $-1,325$ \\
\hline
\end{tabular}

Notas: (a) Los valores de $G S$ y la medida de eficiencia vienen estandarizados.

(b) La media y la desviación estándar de $G S$ son 28,400 y 3,279 respectivamente. Las del índice de eficiencia en $R A P$ y $R A G$ conjuntamente son 0,985 y 0,02 .

Fuente: Elaboración propia a partir de datos de Eurostat, 2018.

\section{Conclusiones.}

En este trabajo evaluamos la eficiencia del gasto social en la reducción de los índices de vulnerabilidad de los países de la EU15 combinando la metodología Data Envelopment Analysis (DEA), que permite medir el grado de eficiencia de los países, con un análisis clúster borroso para detectar patrones en el binomio volumen de gasto social/eficiencia del gasto social. Este análisis nos permite identificar si la efectividad del gasto social tiene relación con la clasificación comúnmente aceptada de Estados de Bienestar (Esping-Andersen, 1990; Ferrera, 1998; 2005): nórdico, continental, anglosajón y mediterráneo. 
En general, los países con modelo de EB nórdico suelen ser eficientes tanto en la reducción de la pobreza como en la de la desigualdad. La única excepción sería Suecia y, en mucho menor grado, Dinamarca que se alinean de forma clara en el primer estado y más residual en el segundo, en el grupo de países escasamente eficientes respecto a la reducción de la pobreza. Los países con EB continental suelen ser eficientes en la reducción de los índices de vulnerabilidad, pero se observan más excepciones que en el caso de los países nórdicos, sobre todo en la reducción de la pobreza. Así, Bélgica, Alemania $\mathrm{y}$, de forma no tan clara, Austria, se incluyen en el grupo de los países no eficientes en la reducción del riesgo de pobreza. En cuanto a la disminución del índice de Gini, Francia y Alemania se alinean en el grupo de países escasamente eficientes. No obstante, cuando consideramos la eficiencia en la reducción de la pobreza y la desigualdad conjuntamente, ningún país con EB nórdico o continental se agrupa en el clúster de estados no eficientes.

Podemos observar que el grupo de países mediterráneos (Italia, España, Grecia y Portugal) se manifiestan consistentemente ineficientes con independencia de que realicemos el análisis en la reducción de la pobreza, de la reducción del índice de Gini o de la reducción de ambos conjuntamente. Forman, de hecho, el núcleo del grupo de países ineficientes en los tres análisis clúster realizados. El comportamiento de los dos países con estado de bienestar anglosajón, Irlanda y Reino Unido, es contradictorio. Irlanda se manifiesta claramente eficiente en la reducción de todos los índices de vulnerabilidad mientras que Reino Unido se agrupa en todos los análisis clúster con los países con EB mediterráneo.

\section{Referencias}

Afonso, A., \& Aubyn, M.S. (2006). Cross-country efficiency of secondary education provision: A semiparametric analysis with non-discretionary inputs. Economic modelling, 23(3), 476-491.

Afonso, A., \& St Aubyn, M. (2004). Non-parametric approaches to education and health expenditure efficiency in OECD countries. Journal of Applied Economics, 8(2), 227-246.

Afonso, A., Schuknecht, L., \& Tanzi, V. (2010). Income distribution determinants and public spending efficiency. The Journal of Economic Inequality, 8(3), 367-389.

Agasisti, T., \& Johnes, G. (2010). Heterogeneity and the evaluation of efficiency: the case of Italian universities. Applied Economics, 42(11), 1365-1375.

Andersson, C., Antelius, J., Månsson, J., \& Sund, K. (2017). Technical efficiency and productivity for higher education institutions in Sweden. Scandinavian journal of educational research, 61(2), 205223.

Anker, J. et al. (2009). Overview and Analysis. Minimum Income Schemes in Denmark: A Study of National Policies, Brussels: European Commission.

Atkinson, A. (2000). A European Social Agenda: Poverty Benchmarking and Social Transfers. Euromod Working Paper No. EM3/00.

Banker RD, Charnes A., \& Cooper WW. (1984). Some models for estimating technical and scale inefficiency in data envelopment analysis. Management Science, 30(9), 1078-1092.

Beblo, M., \& Knaus, T. (2001). Measuring Income Inequality in Euroland. The Review of Income and Wealth, 47, 301-320. 
Bezdek, J.C. (1981). Objective Function Clustering. In Pattern recognition with fuzzy objective function algorithms, Springer, Boston, MA. pp. 43-93.

Bogdanov, G., \& Zahariev, B. (2009). Analysis of the Situation in Relation to Minimum Income Schemes in Bulgaria. A Study of National Policies. Brussels: European Commission.

Bradbury, B., \& Jäntti, M. (2001). Child poverty across twenty-five countries. In Bradbury, B., Jenkins, S., \&Micklewright, J. (eds.), The dynamics of Child Poverty in Industrialised Countries, Cambridge: Cambridge University Press, pp. 62-91.

Cantillon, B., Marx, I., \& Van den Bosch, K. (1997). The Challenge of Poverty and Social Exclusion. In OECD (ed.), Towards 2000: The New Social Policy Agenda, Paris: OECD.

Cantillon, B. (2011). The Paradox of the Social Investment State. Growth, Employment and Poverty in the Lisbon Era. Working Paper No. 11 / 03, University of Antwerp. http://www.centrumvoorsociaalbeleid.be/sites/default/files/CSB\%20Working\%20Paper\%2011\%2 003 March\%202011.pdf

Cantillon, B., Marx, I., \& Van den Bosch, K. (2002). The Puzzle of Egalitarianism: About the Relationships between Employment, Wage Inequality, Social Expenditures and Poverty. Working Papers No 337, Luxembourg: Luxembourg Income Study.

Charnes, A., Cooper, W.W., \& Rhodes, E. (1978). Measuring the efficiency of decision making units. European Journal of Operational Research, 2(6), 429-444.

Cincinnato, S., \&Nicaise, I. (2009). Minimum Income Schemes: Panorama and Assessment. A Study of National Policies, Brussels: European Commission.

Clements, B. (2002). How efficient is education spending in Europe? European Review of Economics and Finance, 1(1), 3-26.

Coccia, M., Falavigna, G., \& Manello, A. (2015). The impact of hybrid public and market-oriented financing mechanisms on the scientific portfolio and performances of public research labs: a scientometric analysis. Scientometrics, 102(1), 151-168.

Costea, A., \& Bleotu, V. (2012). A new fuzzy clustering algorithm for evaluating the performance of non-banking financial institutions in Romania. Economic Computation and Economic Cybernetics Studies and Research, 46, 179-199.

Dave, R.N. (1996). Validating fuzzy partitions obtained through c-shells clustering. Pattern Recognition Letters, 17(6), 613-623.

Derrig, R.A., \& Ostaszewski, K.M. (1995). Fuzzy techniques of pattern recognition in risk and claim classification. Journal of Risk and Insurance, 62(3), 447-482.

Emrouznejad, A., \& Yang, G.L. (2018). A survey and analysis of the first 40 years of scholarly literature in DEA: 1978-2016. Socio-Economic Planning Sciences, 61, 4-8.

Esping-Andersen, G. (1990). Three Worlds of Welfare Capitalism, Cambridge: Cambridge Polity Press. 
Ferrera, M. (2005). Welfare states and social safety nets in Southern Europe: an introduction. In M. Ferrera (Ed.), Welfare State Reform in Southern Europe. Fighting poverty and social exclusion in Italy, Spain, Portugal and Greece, Routledge: Abingdon, pp. 1-23.

Ferrera, M. (1998): Le trappole del welfare, Bolonia: Il Mulino.

Finn, D., Schulte, B., Eichhors, O., Kaufmann, O., \& Konle-Seidl, R. (2008). Employment First': activating the British Welfare State. Bringing the Jobless into Work? Experiences with Activation Schemes in Europe and the US. Berlin: Springer.

González-Pérez, B., Mendaña-Cuervo C., López-González, E., \& Caño-Alegre, C. (2007). ¿Son eficientes las Universidades Públicas? Aplicación del análisis envolvente de datos en el caso de Castilla y León. En P. Moyano Pesquera, N. Somarriba Arechavala, J. E. Fernández Arufe (dir.), J. L. Rojo García (dir.), Anales de economía aplicada 2007, 5, 129-153.

Gupta, S., \& Verhoeven, M. (2001). The efficiency of government expenditure: experiences from Africa. Journal of Policy Modeling, 23(4), 433-467.

Herrera, S., \& Pang, G. (2005). Efficiency of public spending in developing countries: an efficiency frontier approach (Vol. 3645). NY: World Bank Publications.

Hirschinger, M., Spickermann, A., Hartmann, E., Gracht, H., \& Darkow, I.L. (2015). The Future of Logistics in Emerging Markets-Fuzzy Clustering Scenarios Grounded in Institutional and FactorMarket Rivalry Theory. Journal of Supply Chain Management, 51(4), 73-93.

Kandel, A. (1982). Fuzzy Techniques in Pattern Recognition, New York: John Wiley and Sons.

Kapsoli, M.J., \& Teodoru, I.R. (2017). Benchmarking Social Spending Using Efficiency Frontiers. Working Paper 17/197, International Monetary Fund.

Khaleie, S., Fasanghari, M., \& Tavassoli, E. (2012). Supplier selection using a novel intuitionist fuzzy clustering approach. Applied Soft Computing, 12(6), 1741-1754.

Khoshnevisan, B., Rafiee, S., Omid, M., Mousazadeh, H., Shamshirband, S., \& Ab Hamid, S. H. (2015). Developing a fuzzy clustering model for better energy use in farm management systems. Renewable and Sustainable Energy Reviews, 48, 27-34.

Klawonn, F., Kruse, R., \& Winkler, R. (2015). Fuzzy clustering: More than just fuzzification. Fuzzy sets and systems, 281, 272-279.

Legros, M. (2009). Minimum Income Schemes. From Crisis to Another, The French Experience of Means-tested Benefits, Brussels: European Commission.

Liu, J. S., Lu, L. Y., Lu, W. M., \& Lin, B. J. (2013). A survey of DEA applications. Omega, 41(5), 893902.

Nelson, K. (2003). Fighting Poverty: Comparative Studies on Social Insurance, Means-tested Benefits and Income Redistribution. Dissertation Series No. 60, Stockholm: Swedish Institute for Social Research.

Oxley, H., Dang, Th.-Th., Förster, M., \& Pellizari, M. (2001). Income inequalities and poverty among children and households with children in selected OECD countries. In K. Vleminckx y T. Smeeding 
(eds), Child Well-being, Child Poverty and Child Policy in Modern Nations: What do we know, Bristol: Policy Press, pp. 371-405.

Radu, M. (2009). Analysis of the Situation in Relation to Minimum Income Schemes in Romania. A Study of National Policies. Brussels: European Commission.

Ruoppila, S., \& Lamminmäki, S. (2009). Minimum Income Schemes. A Study of National Policies. Brussels: European Commission.

Sherman, H. D., \& Zhu, J. (2006). Data Envelopment Analysis Explained. Service Productivity Management: Improving Service Performance using Data Envelopment Analysis (DEA), 49-89.

Vandenbroucke, F., \& Vleminckx, K. (2011). Disappointing poverty trends: is the social investment state to blame? An exercise in soul-searching for policy-makers. Working Paper No. 11 / 01, University of Antwerp.

Wang, W., \& Zhang, Y. (2007). On fuzzy cluster validity indices. Fuzzy sets and systems, 158(19), 2095-2117.

Wu, D., Yan, D.H., Yang, G.Y., Wang, X.G., Xiao, W.H., \& Zhang, H.T. (2013). Assessment on agricultural drought vulnerability in the Yellow River basin based on a fuzzy clustering iterative model. Natural hazards, 67(2), 919-936.

Xie, X.L., \& Beni, G. (1991). A validity measure for fuzzy clustering», IEEE Transactions on pattern analysis and machine intelligence, 13(8), 841-847.

Yu, S., Wei, Y.M., \& Wang, K. (2014). Provincial allocation of carbon emission reduction targets in China: an approach based on improved fuzzy cluster and Shapley value decomposition. Energy Policy, 66, 630-644.

Yu, S., Wei, Y.M., Fan, J., Zhang, X., \& Wang, K. (2012). Exploring the regional characteristics of inter-provincial $\mathrm{CO} 2$ emissions in China: An improved fuzzy clustering analysis based on particle swarm optimization. Applied Energy, 92, 552-556 